\title{
High-Content Imaging to Profile Cellular and Pathogen Responses to Bioactive Probes
}

\author{
R.G. Panchal, ${ }^{*}$ K. Kota, ${ }^{* *}$ D. Lane, ${ }^{* * *}$ Brett Eaton, $*$ and S. Bavari*
}

* Department of Target Discovery \& Experimental Microbiology, United States Army Medical Research Institute of Infectious Diseases, Frederick, MD 21702

** PerkinElmer, Waltham, MA 02451

*** Target Structure Based Drug Discovery Group, SAIC-Frederick, Inc., NCI-Frederick, National Institute of Health, Frederick, MD 21702

In recent years, anti-bacterial drug discovery has met with little success and coupled with the rise in drug resistance in many pathogens, there is an increasing need to identify new targets and new approaches to antimicrobial drug discovery. Screening for novel antibacterials has traditionally been done by scoring for growth inhibition in vitro on artificial media. This approach has over the years led to a limited number of antibacterial compounds. An added disadvantage to this approach is the inability to identify potent antimicrobial compounds for intracellular pathogens that require the host for replication. Hence, there is a need to develop novel therapeutic strategies for antibacterial drug discovery.

It is well established that the interplay between bacteria and host cells determines the outcome of disease pathogenesis, ranging from the elimination of bacteria to latent or lethal infections. Many pathogenic bacteria exploit the host's immune machinery and subsequently modulate cell function, signaling, migration and cytoskeleton rearrangement. Hence, identifying host cellular components with which the microbes interact will allow for a more comprehensive understanding of microbial pathogenesis, define common strategies used by multiple pathogens and elucidate unique tactics evolved by individual species to help establish infections or evade host innate responses. Another interesting aspect of infection is that diverse pathogens seem to target common cellular pathways. Thus, identifying host targets exploited by multiple pathogens will be useful in the development of broad-spectrum host-oriented therapeutics and vaccines. The success in developing therapeutics targeted against the bacterial virulent factors or the host will be dependent on design of innovative high-throughput approaches to study host-pathogen interactions and subsequent screening of small molecule chemical libraries to identify compounds that disrupt the host-pathogen interactions.

Visual insights into host-pathogen interactions are important for the development of hostoriented therapeutics, as it will provide critical information regarding whether the disruption of infection by small molecule therapeutics will result in cellular toxicity, phenotypic changes and/or the modulation of host biological responses. In fact, major advances in optical microscopy combined with the power of automated image processing has led to development of high-content imaging (HCI) assays, to measure multiple cellular responses to drugs or biological stimuli in single cells. This emerging technology has made a significant impact on drug discovery. However, application of this technology for the quantitative measurement of pathogen infected cells or phenotypic alterations of the host cells following pathogen infection is still fraught with logistic and technical difficulties for screening and identifying compounds against deadly pathogens.

In this study, we report the development and optimization of HCI assays to quantitate pathogen uptake or phagocytosis, intracellular bacterial replication, modulation of host signaling events, and induced changes in host cell morphology following infection of macrophages with the 
virulent Category A/B bacteria. Subsequent screening of pharmacologically active small molecules in the HCI assays, identified compounds that either inhibited or potentiated one or more of the microbiological phenotypes. Thus, image-based assays in conjunction with chemical genetic screening have led to the identification of host cell targets and cellular pathways that are involved in the host-pathogen interactions.

This research was supported by the Defense Threat Reduction Agency under contract 2.10019_09_RD_B to RGP. The aid of Dr. Dutch Boltz, Perkin Elmer is gratefully acknowledged.

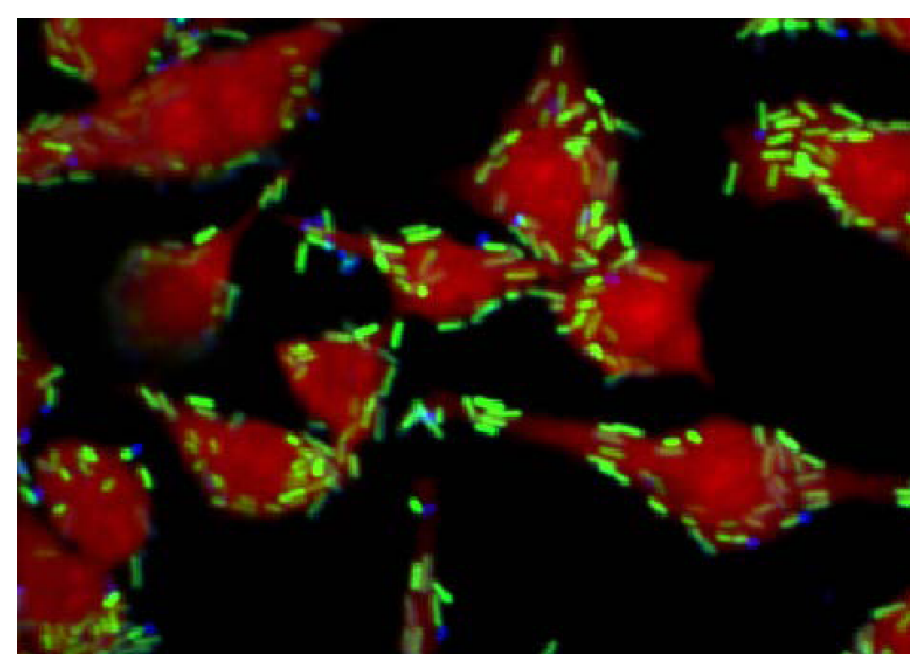

FIG.1. Macrophages infected with GFP expressing Bacillus anthracis. Cells are stained with cell mask deep red that stains the cytoplasm of the cell. Bacteria pseudo colored blue/cyan are outside of the cell, as visualized by staining of non-permeabilized infected cells with antibacterial antibody. 